\title{
Sur les bords de la protestation sociale.
} Illustrations sud-africaines

At the Edges of Social Protest. Illustrations from South Africa

Jérôme Tournadre

\section{(2) OpenEdition \\ Journals}

Édition électronique

URL : http://journals.openedition.org/conflits/19191

DOI : 10.4000/conflits. 19191

ISSN : $1777-5345$

Éditeur :

CCLS - Centre d'études sur les conflits lilberté et sécurité, L'Harmattan

Édition imprimée

Date de publication : 19 mai 2016

Pagination : 121-138

ISBN : 978-2-343-09459-5

ISSN : 1157-996X

Référence électronique

Jérôme Tournadre, "Sur les bords de la protestation sociale. Illustrations sud-africaines », Cultures \& Conflits [En ligne], 101 | printemps 2016, mis en ligne le 19 mai 2017, consulté le 30 mars 2021. URL: http://journals.openedition.org/conflits/19191 ; DOI : https://doi.org/10.4000/conflits.19191 


\section{Sur les bords de la protestation sociale. Illustrations sud-africaines}

\section{Jérôme TOURNADRE 1}

Jérôme Tournadre est chargé de recherche CNRS à l'Institut des sciences sociales du politique (CNRS/Université Paris Ouest/ENS Cachan). Ses travaux portent sur l'histoire sociale des idées politiques contemporaines dans le monde anglo-saxon et sur la protestation sociale en Afrique du Sud postapartheid. Il a notamment publié $\mathrm{Au}$-delà de la gauche et de la droite, une troisième voie britannique ? (Dalloz, 2006), Après l'apartheid (PUR, 2014) et, avec Arnault Skornicki, La Nouvelle histoire des idées politiques (La Découverte, 2015).

T'analyse des mouvements sociaux serait trop étroitement balisée, à en croire les appels relativement récents et explicites à «élargir notre conceptualisation [...] au-delà de la politique contestataire 2 ». À ceux-ci, ont semblé répondre diverses tentatives en vue de placer sous l'étendard mouvementiste des formes d'actions collectives qui en avaient jusqu'alors été écartées ${ }^{3}$. C'est cependant une autre façon de concevoir l'élargissement que l'on trouvera au principe de ce texte, à distance de cette tendance à concentrer l'étude sur les seules interactions avec les autorités politico-administratives ${ }^{4}$. Cette réflexion s'appuie sur les résultats d'une enquête conduite depuis 2009 dans différentes agglomérations sud-africaines (Johannesburg, Le Cap, Durban et

1. Ce texte doit beaucoup aux commentaires de Florence Johsua, Sophie Duchesne, Elisabeth Claverie, Nathalie Duclos, Milena Jaksic, Nicole Kerchen, Eric Phélippeau, Clotilde Riotort, Carole Sigman et Arnault Skornicki, ainsi qu’à ceux des deux lecteurs anonymes de la revue.

2. Snow D., "Social movements as challenges to authority: Resistance to an emerging conceptual hegemony ", in Myers J. M., Cress D. M. (eds.), Authority in Contention (Research in Social Movements, Conflicts and Change, Volume 25), Bingley, Emerald Group Publishing Limited, 2004, pp. 3-25.

3. À l'instar de «styles de vie » comme le véganisme ou la vie communautaire, si l'on suit Haenfler R., Johnson B., Jones E., « Lifestyle Movements: Exploring the Intersection of Lifestyle and Social Movements ", Social Movements Studies, vol. 11, n 1, janvier 2012, pp. 120.

4. Sur la critique de cette tendance, voir Van Dyke N., Soule S. A., Taylor V. A., « The Targets of social movements: Beyond a focus on the State ", in Myers J. M., Cress D. M. (eds.), op. cit., pp. 27-51 ; également Agrikolianski É., Dufour P., « Les frontières des mouvements sociaux/Les mouvements sociaux aux frontières ", Politique et Sociétés, vol. 28, $\mathrm{n}^{\circ}$ 1, 2009, p. 3-11. 
Grahamstown), au contact de militants actifs dans des organisations qui, pour certaines depuis une quinzaine d'années, donnent forme au mécontentement généré par le manque de logements et l'accès défaillant aux réseaux d'eau, d'électricité et d'assainissement ${ }^{5}$. La rareté de ces biens fondamentaux, pourtant au cœur des promesses et des politiques mises en place par les gouvernements depuis les premières élections démocratiques ${ }^{6}$, est en effet l'un des aspects les plus saillants d'une situation sociale où plus de la moitié de la population vit actuellement sous le seuil de pauvreté absolue ${ }^{7}$.

De façon somme toute classique, ces recherches reposent sur la conduite d'entretiens et d'observations ; observations de réunions publiques et d'actions protestataires (c'est-à-dire de périodes où les organisations sont porteuses de revendications) mais également de moments en apparence plus anodins, comme par exemple ces heures passées sans but réel dans les locaux des organisations, à écouter les conversations les plus diverses entre militants et habitants du quartier, ou à accompagner les premiers dans la résolution de conflits de voisinage. À chaque fois, c'est bien le collectif protestataire que j'observe, ses militants continuant, même dans le cadre de ces activités, de s'en revendiquer. Mais à la différence de ce que je peux saisir le reste du temps, leur parole et leur regard ne sont plus tournés vers le pouvoir politique. On pourrait dire, en empruntant certains raccourcis, que la protestation n'est alors plus seulement la protestation ou, à tout le moins, qu'elle semble s'écarter des définitions consacrées.

Je n'entends pas, dans les pages qui suivent, remettre en cause lesdites définitions, l'objet étudié - différents collectifs protestataires sud-africains étant a priori conforme aux canons de la sociologie des mouvements sociaux. Il s'agit plutôt d'esquisser une analyse débordant le cadre qu'elles contribuent à dessiner et, en s'éloignant ainsi des dimensions essentiellement «politiques » de la protestation, de mettre en relief des facettes parfois moins visibles des organisations et du travail militants. C'est en cela que l'on peut prétendre élargir un peu le spectre de l'étude : en insistant sur le fait que l'on ne voit et que l'on ne comprend pas tout si l'on s'en tient à ces « moments où les gens s'assemblent pour faire des revendications vigoureuses, visibles, publiques, agissent à partir de ces revendications d'une manière ou d'une autre, puis retournent à d'autres affaires ${ }^{8}$ ». Au tête à tête bruyant avec les autorités politiques

5. Les entretiens ont principalement été réalisés entre juillet 2009 et avril 2015 avec des militants $\mathrm{du}$ Soweto Electricity Crisis Committee (SECC), du Thembelible Crisis Committee (TCC), de l'Anti-Privatisation Forum (APF), de l'Unemployed People's Movement (UPM) et d'Abablali base Mjondolo (AbM).

6. L'African National Congress (ANC) domine tous les scrutins depuis 1994 et gouverne officiellement avec ses anciens partenaires de la lutte contre l'apartheid : le Parti communiste et la confédération syndicale COSATU.

7. StatsSA, Methodological report on rebasing of national poverty lines and development of pilot provincial poverty lines, Statistics South Africa, Pretoria, 2015.

8. Tilly C., "Contentious repertoires in Great Britain, 1758-1834 », in Traugott M. (ed.), 
ou au bourdonnement des revendications sera par conséquent préféré un (ré)encastrement de la protestation et de ses acteurs dans leur environnement social et spatial le plus immédiat, celui que composent les quartiers populaires sud-africains : les parties les plus modestes des townships et les zones d'habitat informel. Les collectifs protestataires apparaissent alors immergés dans un certain ordinaire dont le mérite principal est de rappeler que le mécontentement organisé n'est pas un objet hors-sol : il s'inscrit dans des tissus de relations sinon "normales 9 ", à tout le moins banales ou routinières, cet état de fait étant d'autant plus marqué dans le cas sud-africain que les quartiers populaires y sont tout à la fois le théâtre des manifestations et le lieu vécu ${ }^{10}$ des femmes et des hommes qui y prennent part. Apparaîtront alors certains des liens, parfois très continus, entre la protestation et ce qui constitue la principale temporalité de cet ordinaire, celle du quotidien. Si elle n'est pas totalement absente des travaux sur les mouvements sociaux ${ }^{11}$, l'idée qu'il puisse exister autre chose qu'une césure entre protestation et vie quotidienne semble régulièrement écartée par un certain sens commun (mais il n'est pas le seul). Un fossé s'insinuerait immanquablement entre cette sphère emplie du bruit irruptif des cortèges et la trame du commun, du régulier et de l'habituel. Peutêtre est-ce pourtant dans ces rapports au quartier, dans certains plis du quotidien ou, pour reprendre l'expression de Charles Tilly, dans ces « autres affaires » auxquelles vaquent les militants quand ils ne protestent pas, que se nichent des éléments éclairant quelques-unes des logiques ou pratiques structurant la mobilisation ${ }^{12}$; des éléments qui traduisent, pour certains, un rapport ordinaire au politique, voire des formes d'infrapolitique ${ }^{13}$, et qui, pour d'autres, n'apparaissent pas comme fondamentalement politisés ou politisants.

\section{Une protestation située}

La protestation sociale sud-africaine ne s'aventure que rarement au-delà des limites physiques des zones pauvres des townships ou des ruelles sinuant entre les cabanes en tôle et en matériaux de récupération divers qui dessinent les espaces de logements informels. Mais en plus d'accueillir des marches pacifiques, des barrages d'axes routiers et autres invasions de logements fraîchement sortis de terre, les quartiers populaires sont très souvent le seul horizon physique des femmes et des hommes qui, depuis une quinzaine d'années, participent à la mise en forme du mécontentement social. Pour des raisons qui se

Repertoires \& Cycles of collective action, Durham et Londres, Duke University Press, 1995, p. 32 .

9. Auyero J., "L'espace des luttes. Topographie des mobilisations collectives ", Actes de la recherche en sciences sociales, $\mathrm{n}^{\circ} 160,2005$, p. 128.

10. Bonny Y., Ollitrault S., Keerle R. et Le Caro Y., Espaces de vie, espaces enjeux. Entre investissements ordinaires et mobilisations politiques, Rennes, Presses universitaires de Rennes, 2011.

11. Voir cependant Auyero J., "When everyday life, routine politics, and protest meet », Theory and Society, vol. 33, n ${ }^{\circ}$ 3, 2004, pp. 417-441.

12. Cette citation de Tilly ne me conduit pas à oublier combien les « répertoires d'action » ne sont pas sans lien avec certains aspects de la vie quotidienne.

13. Scott J. C., La Domination et les arts de la résistance, Paris, Éditions Amsterdam, 2008. 
nichent au croisement d'une géographie héritée de l'apartheid ("séparation » en Afrikaans) et de leur propre condition socio-économique, ces populations sortent peu de ces espaces. La faute aux politiques ségrégatives et racistes, qui reléguèrent les non-Blancs en périphérie des villes, parfois (notamment dans les grandes agglomérations) à plusieurs dizaines de kilomètres des centres urbains, et à une démocratie qui n'a pas encore remédié à cette situation. Pour sortir de ces espaces, les habitants les plus pauvres doivent ainsi s'en remettre à de médiocres services de bus ou à des taxis collectifs dont le coût s'avère souvent dissuasif.

L'ancrage de la protestation dans ces zones d'habitations (formelles et informelles) trouve un premier relais dans les formes organisationnelles que celle-ci a privilégiées depuis la fin des années 1990. Le plus souvent, ces collectifs adoptent le modèle du parapluie (on parle ainsi d'umbrella organisations) : un premier étage, constitué au niveau d'une ville, d'une agglomération ou d'une région, coordonne l'action d'associations qui lui sont affiliées et dont l'activité est repérable au niveau d'une township, d'un camp de squatters, d'un quartier ou d'un voisinage. Ces affiliés s'apparentent à des regroupements d'habitants autour de quelques militants très investis et visibles dans leur communauté. On retrouve ici un type de structure que connaissent assez bien celles et ceux qui vivent dans ces zones d'habitat modeste ou pauvre. Le maillage social de ces dernières est, en effet, régulièrement parcouru par diverses associations (comités de "résidents concernés » ou comités « de crise », mais aussi associations sportives, collectifs de retraités, comités de vigilance, stokvels ${ }^{14}$, etc.) dont on relève les premières traces à la fin du XIX ${ }^{\mathrm{e}}$ siècle ${ }^{15}$, dans certains quartiers noirs. Cette forme associative connaît un nouveau souffle dans les années 1970, au travers du mouvement des civics ${ }^{16}$, enjoint par les dirigeants en exil de l'African National Congress (ANC) de "rendre les townships ingouvernables ». Les organisations protestataires contemporaines recourent donc à des modèles de groupement relativement routinisés, quand elles ne prennent pas directement le relais d'associations ou de groupes préexistants. À Mandela Park, dans l'agglomération du Cap, la représentation locale de l'Anti-Eviction Campaign (AEC) ${ }^{17}$ qui se met en place lors de la première moitié des années 2000 est ainsi un décalque du comité de vigilance local qui officiait quelques années auparavant. À Isipingo, près de Durban, l'une des branches du Concerned Citizens' Forum est, à la même époque, créée

14. Les stokvels sont de petites associations collectives d'épargne rappelant le modèle de la tontine.

15. Bundy C., "Survival and Resistance: Townships Organisations and Non-violent direct Action in Twentieth Century ", in Adler G., Steinberg J. (eds.), From Comrades to Citizens. The South African Civics Movement and the Transition to Democracy, Londres, Palgrave Macmillan \& Albert Einstein Institute, 2000, pp. 26-51.

16. Les civics sont des regroupements d'habitants au niveau d'une rue ou d'un quartier.

17. Luttant notamment contre les expulsions frappant les ménages pauvres de l'agglomération du Cap, l'AEC était l'une des organisations les plus visibles dans l'espace public au cours des années 2000. 
par un instituteur syndiqué qui réactive les réseaux du civic devenu moribond dans son quartier. Un effort est même parfois ostensiblement consenti pour inscrire le nouveau collectif dans cette tradition et dans l'histoire du territoire, comme le suggèrent les propos de l'un des fondateurs du Soweto Electricity Crisis Committee (SECC) :

"Nous avons donc décidé de fonder cette organisation, puis nous l'avons appelée le Soweto Electricity Crisis Committee parce que, à Soweto, il y avait une organisation, durant le combat contre l'apartheid, dans les années 1980, qui s'appelait le Soweto Education Crisis Committee et qui était très forte dans le rassemblement des étudiants et des parents contre l'apartheid. [Et des gens de cette première organisation ont rejoint le Soweto Electricity Crisis Committee ?] Non, non. C’était juste pour la mémoire ( $\mathrm{Nda}$ : il porte la main droite au niveau de sa tempe, pour suggérer la réflexion). [Pour marquer un lien avec la lutte ?] Oui, oui. Ca s'appelait le Soweto Education Crisis Committee, donc nous avons dit: "Nous sommes le Soweto Electricity Crisis Committee". " (Entretien, 7 juillet 2009, Durban)

Les collectifs protestataires contemporains émergent donc d'une structuration sociale qui leur préexiste et peuvent a priori en tirer quelque bénéfice. Cette situation leur assure, très souvent, un accès à certaines ressources dont on sait qu'elles sont essentielles au passage à l'action 18 : un local de réunion, des meneurs rompus aux méthodes d'organisation ou, plus encore, un groupe d'individus déjà constitué. Ce dernier élément apparaît d'ailleurs central dans la formation des organisations protestataires contemporaines ou, plus précisément, dans la composition de leur noyau militant originel. À Orange Farm, en périphérie de Johannesburg, le rassemblement d'habitants au sein de l'Orange Farm Water Crisis Committee est orchestré par des femmes et des hommes ayant quitté les sections locales de l'ANC et de SANCO ${ }^{19}$ pour dénoncer l'inaction de leurs responsables face aux coupures d'eau et d'électricité récurrentes depuis 1996. L'interconnaissance joue également un rôle important par la suite. Ceux qui rejoignent les organisations après leur formation et en composent la base sociale lorsqu'ils prennent part, plus ou moins ponctuellement, aux marches ou aux rassemblements, ont souvent découvert le collectif par le biais d'un ami ou d'un voisin qui en était déjà membre, ou après avoir accompagné un proche dans l'une des nombreuses réunions publiques animées par des activistes. Le cas de ces rassemblements est d'ailleurs intéressant à plus d'un titre. Se tenant parfois dans le froid ou à la nuit tombante ${ }^{20}$, à la lisière de

18. McAdam D., Political Process and the development of Black Insurgency (1930-1970), Chicago, The University of Chicago Press, 1999 (1982), pp. 44-48.

19. Proche des groupes dirigeants de l'ANC, la South African National Civic Organisation (SANCO) fédère un grand nombre de civics depuis 1992.

20. Observation, Grahamstown, avril 2014. 
zones d'habitations ou dans des centres communautaires de quartiers, ils sont ouverts à tous les habitants, qui s'y rendent parfois avec des enfants en bas âge. La parole est censée y être libre; elle circule et se concentre sur des problèmes très locaux. Les dirigeants nationaux de l'ANC y sont d'ailleurs rarement dénoncés par les militants, les critiques se concentrant en général sur les élus municipaux. Il peut d'ailleurs arriver que des militants cherchent à rassurer les habitants présents en les assurant que la réunion ne sera pas consacrée à « la politique mais aux bread-and-butter issues $21 »$. Les protestataires, qui voient dans ces moments autant d'occasions de convaincre de la nécessité de rejoindre la cause, peuvent en outre y rappeler les « succès » passés de leur organisation : l'installation par la municipalité de toilettes dans un camp de cabanes, l'obtention d'un moratoire sur les déconnexions à l'électricité, etc. Certes, dans ces moments, les revendications resurgissent, la trahison des espoirs de 1994 est - même implicitement - dénoncée, les élus locaux sont critiqués pour leur implication dans le sous-développement du quartier, et l'on rejoint donc a priori les définitions classiques du mouvement social. Mais le plus important est peut-être moins ce que sont ces pratiques et ces discours que ce qu'ils sont censés être : insérés dans une forme politique pleinement maîtrisée par les habitants et en phase avec leurs maux quotidiens ; une politique que l'on pourrait presque qualifier d'élémentaire, car aux avant-postes de ce qui serait essentiel et fondamental: une "politique populaire».

\section{Une politique populaire?}

Exprimer son mécontentement en se lançant dans un toyi toyi ${ }^{22}$, se rendre aux assemblées réunies pour discuter des problèmes menaçant le quartier, participer à l'élection de leaders communautaires, appartenir à des comités de voisinage... Autant d'éléments constitutifs de cette forme politique dont la spécificité serait de s'inscrire directement dans le quotidien du quartier. Le terme même de « politique populaire » doit certainement plus à un travail de conceptualisation intellectuelle et universitaire qu'aux mots de celles et de ceux qui en sont les premiers acteurs et témoins. Il est en outre peu probable que ces derniers accordent systématiquement une dimension politique à ce qu'ils font alors tous les jours (ou presque). Certains de leurs actes, comme le fait de se rendre à une assemblée d'habitants, peuvent même être effectués sans conviction, par conformisme social 23 ou au nom d'une habitude dont les fondements ne sont guère interrogés : parce que cela va de soi ou parce que « tout le monde le fait ». Mais au-delà de quelques nécessaires précautions d'emploi, il ne fait a priori aucun doute que cette catégorie - la «politique populaire »-

21. Observation, Soweto, avril 2015.

22. Le toyi-toyi mêle danse, slogans politiques et chants de combat ou de résistance. Il était régulièrement pratiqué durant l'apartheid lors des manifestations contre la politique du gouvernement.

23. Voir Mariot N., Bains de foule. Les voyages présidentiels en province, 1888-2002, Paris, Belin, 2006. 
rassemble des activités, des moments, des récits, des discours, des prescriptions et des représentations ayant en commun de prétendre à la régulation et au gouvernement de la vie locale. Cet ensemble est par ailleurs donné à voir comme une rencontre harmonieuse entre social et politique. À titre d'exemple, les réunions que le SECC tient chaque semaine dans les locaux ou la cour du Careers Centre de Diepkloof (Soweto) s'achèvent parfois sur des offres de partage de connaissance ou de maîtrise d'une technique ou d'un savoir pratique : l'un des membres de l'assistance, qu'il soit simple habitant venu se plaindre ou militant, peut profiter de ce moment pour proposer des cours de couture ou de mécanique à celles et ceux qui, quelques minutes auparavant, s'indignaient des coupures d'électricité décidées par la société parapublique.

Une simple mise en perspective vient par ailleurs rappeler qu'une « culture est historique avant d'être “culturelle” 24 ». La politique populaire dispose en effet d'une certaine profondeur historique, celle de ces sociabilités de voisinage qui, dès la fin du XIX ${ }^{\mathrm{e}}$ siècle, apparaissent dans les mondes noirs. L'institutionnalisation de la ségrégation et l'interdiction formelle faite aux non-Blancs de participer à la vie politique officielle l'ont, en outre, renforcée et actualisée au milieu du siècle suivant. Elle a alors pu se confondre avec le principe du «pouvoir populaire » (people's power) et sa logique de contournement des institutions imposées par l'apartheid, et se cristalliser autour de croyances spécifiques, dont celle d'être façonnée par et pour les habitants.

Tous ces éléments font en outre l'objet d'une mise en cohérence et d'un entretien régulier par des groupes se posant en gardiens de la politique populaire : des leaders communautaires, des membres des nombreux comités de rue, de quartier et de civics ou, désormais, des militants protestataires. Tous n'ont de cesse d'actualiser ces activités, de leur donner du sens et, plus encore, de les présenter comme les fondements d'une tradition politique parfaitement et durablement assimilée par les habitants ; à la différence de l'autre politique, celle qui se pratique dans le monde de la démocratie libérale. Au début des années 1990, alors que l'apartheid rendait son dernier souffle, les propos de certains leaders communautaires en disaient d'ailleurs toute la centralité. À la veille des premières élections locales démocratiques, n'affirmaient-ils pas que ces dernières n'étaient "d'aucune utilité, les civics constituant déjà une forme démocratique de gouvernement 25 » ? La frontière est cependant plus « indistincte 26 » qu'annoncé entre une forme politique prisée du « peuple des townships » et celle de gouvernants garants du politique institué depuis 1994. D’une part, les seconds, c'est-à-dire principalement les responsables de l'ANC, ont souvent grandi dans les zones ouvrières ou pauvres des townships et ont été en

24. Bayart J.-F., «Le politique par le bas en Afrique noire. Questions de méthode », Politique africaine, $\mathrm{n}^{\circ} 1$, mars 1981, p. 57.

25. Friedman S., Reitzes M., Democratic Selections? State and Civil Society in Post-Settlement South Africa, Midrand, Development Bank of South Africa, 1995.

26. Bayart, art. cit., p. 57. 
partie socialisés par ce qui ferait la spécificité de la politique populaire. Ils ne manquent d'ailleurs pas de faire valoir cet aspect de leur identité politique, notamment lorsque, au nom d'un "retour aux fondamentaux » (back to basics), ils en insèrent certains éléments dans leurs propres approches de la démocratie libérale. Que l'on pense à l'organisation d'imbizos 27 lors des dernières campagnes électorales de l'ANC. D'autre part, on assiste, depuis la première moitié des années 1990, à l'appropriation de certains pans de la politique populaire par le pouvoir politico-administratif, qui les institutionnalise. Il en va ainsi de sa dimension participative et de sa propension à développer des espaces de discussion, comme en atteste la multiplication des structures (community policing forum, development forum, RDP 28 forum, ward committee, etc.) censées permettre un dialogue entre gouvernants et gouvernés autour de préoccupations locales (sécurité, logement, etc.) ${ }^{29}$.

\section{Servir la communauté}

Les organisations œuvrent avant tout à susciter et à encadrer la mobilisation des habitants de ces zones pauvres d'Afrique du Sud. Mais une analyse du militantisme au quotidien ${ }^{30}$ permet d'entrevoir un autre type de rapport au quartier et à sa population. Celui-ci se déploie en effet dans des choses peu visibles car moins spectaculaires qu'une manifestation. Il s'agit, principalement, de toute une série de services plus ou moins conséquents. Le principe même du service n'est, il est vrai, pas absent de ce qui constitue le cœur de la pratique protestataire, et en premier lieu des reconnexions aux réseaux d'eau et d'électricité de ménages n'ayant pas honoré leurs factures. À Soweto, durant les années 2000, les militants raccordent régulièrement au gré des demandes individuelles : des habitants contactent directement la permanence de l'organisation ou se rendent à l'une des réunions publiques animées par les activistes afin de faire part de leur situation de « débranchés ». En réponse, des militants-électriciens sont dépêchés chez eux et tentent de remédier au problème à l'aide d'un matériel de fortune. Ils endossent alors le risque inhérent à l'illégalité d'une telle pratique.

«Tout le monde se connecte ou se reconnecte et personne ne paie. On [le SECC] continue de connecter car on symbolise la connexion. Tout le monde le fait ou voudrait le faire. Mais les gens ont besoin d'une couverture, donc ils viennent nous voir ». (Entretien avec l'organisateur du SECC, Durban, 7 juillet 2009)

27. L'imbizo est une assemblée où le peuple discute avec les leaders traditionnels.

28. Manifeste électoral de l'ANC en 1994 puis feuille de route des premiers gouvernants démocratiques, le Reconstruction and Development Programme (RDP) prévoyait de réparer les injustices de l'apartheid au travers d'engagements en matière de santé, d'éducation, de raccordement aux services domiciliaires en réseaux et de construction de logements.

29. On peut aussi y voir la trace d'un discours sur le développement particulièrement en vogue dans le monde des années 1990, où la participation apparaissait comme l'un des piliers d'une «bonne gouvernance » misant sur la décentralisation. 
La contrepartie de cette prise en charge du risque est une certaine " politisation de la quotidienneté 31 ». Le boycott des factures ou le rebranchement sauvage sont ainsi totalement mis en sens par de nombreux acteurs de la protestation, qui en font autant d'actes d'une résistance directe et uniformément politique à l'agression dont se rendraient coupables les gouvernants 32 .

Mais au-delà, il est des services, a priori plus anodins et à mille lieues des mots d'ordre de la contestation, qui contribuent à tisser et à entretenir un lien entre les collectifs protestataires et la population. La connexion internet, la ligne téléphonique ou le maigre matériel informatique d'une organisation sont parfois mis à la disposition de voisins ou d'habitants du quartier qui en sont dépourvus. C'est manifestement dans ce cadre que la voiture du chercheur étranger que je suis acquiert, à son tour, le statut de ressource. Il m'est ainsi régulièrement demandé, lorsque j'accompagne des militants sur le lieu d'un rassemblement, de faire monter dans le véhicule une, deux ou trois personnes dont je comprends qu'elles sont extérieures à l'organisation mais font partie des connaissances (plus ou moins vagues) de l'un des activistes, puis de faire un détour pour les déposer chez elles ou à proximité d'un commerce quelconque. Dans un même ordre d'idées, les militants peuvent apporter une aide ponctuelle à des ménages incapables de remplir un document administratif ou désemparés face à la technicité de factures d'eau et d'électricité. Ce sont certainement ces savoirs pratiques supposés qui poussent un jeune habitant de Soweto à se rendre dans les locaux du SECC. Orphelin depuis peu, il craint en effet de ne pouvoir bénéficier des démarches entamées par ses parents pour obtenir une maison RDP et vient donc s'informer auprès des militants ${ }^{33}$. Les militants se prévalent d'ailleurs régulièrement d'une telle connaissance des rouages administratifs, comme le suggère cette militante de Johannesburg détaillant tout l'intérêt qu'il peut y avoir à « collecter des informations sur la situation des gens » dans son quartier, qui lui permettent d'affuter ses « arguments pour [les] faire venir aux réunions » : elle leur explique qu’y sera délivrée l'information dont ils ont besoin pour résoudre certains de leurs problèmes ${ }^{34}$.

Enfin, il ne faut pas négliger ce qui se joue lors de moments aussi routiniers que l'ouverture du local de l'organisation, le matin. Au fil de la journée, les militants qui y sont préposés accueillent, autour d'un thé au rooibos, des habitants venus chercher un renseignement ou, plus simplement, un peu de

30. Neveu É., Sociologie des monvements socianx, Paris, La Découverte, 5e édition, 2011, p. 72.

31. Mouchard D., Etre représenté. Mobilisations d'" exclus " dans la France des années 1990, Paris, Economica, 2009 , p. 88 et s.

32. Cohabitent pourtant dans ces actes, des éléments certes contestataires mais également des impératifs de subsistance ou des réflexes de débrouillardise pour des ménages qui, comme tout un chacun, doivent cuisiner, s'éclairer, se chauffer, se laver (Tournadre J., Après l'apartheid. La protestation sociale en Afrique du Sud, Rennes, PUR, 2014, pp. 115-119).

33. Observation, juillet 2009.

34. Entretien, 14 mai 2011, Thembelihle. 
compagnie. Au-delà de leur contribution au lien social, ces moments révèlent également ce qui fait tenir une organisation : ils favorisent et entretiennent une forme d'attachement (commitment ${ }^{35}$ ) de ces militants à la cause et à l'organisation. Parce qu'ils représentent ces dernières lors de ces interactions en apparence banales, ces individus se voient en effet investis d'un rôle social valorisant. Ces tâches prennent en outre d'autant plus de sens aux yeux de nombre d'entre eux qu'elles sont porteuses d'un sentiment d'intégration aussi structurant que pourrait l'être celui produit par une activité professionnelle :

« Je suis bénévole, je fais ce qu'il faut pour aider l’organisation... Même nettoyer le sol s'il le faut [...] J'aime être ici, parler avec mes camarades... Par exemple, aujourd'hui, je ne suis pas supposé être là... Mais j'aime bien être ici, discuter de politique, de la lutte... » (Entretien, 15 juillet 2009, Johannesburg)

À l'inverse, le quotidien « naturel » du chômeur, celui qui a régulièrement précédé l'investissement dans l'organisation, est bien souvent associé à l'inutilité ( Avant ça, je restais chez moi... Je ne faisais rien »).

Une telle approche, concentrée sur un travail militant que n'épuisent pas les temps forts de la protestation, permet également de mettre en relief des positionnements et des prises de rôles peut-être plus inattendus mais directement rattachés à la vie quotidienne du quartier. C'est ce qu'illustre un certain nombre d'initiatives venant, à première vue, combler les carences de la puissance publique dans les zones pauvres, qu'il s'agisse de la création par des militants de l'AEC d'une école destinée à accueillir les enfants dont les parents ne peuvent payer les droits d'inscription dans le système officiel ou de celle d'une crèche par les membres d'Abablali baseMjondolo Western Cape. Une même forme d'interventionnisme transparaît lorsque les militants de l'Unemployed People's Movement (UPM), à Grahamstown, assurent une médiation entre des ouvriers et leur employeur, organisent des réunions d'information sur les effets néfastes d'un alcool artisanal ou, comme je l'observe en avril 2014, tentent d'obtenir d'un couple qu'il retire la plainte déposée contre la petite amie de leur fils à l'issue d'une violente dispute familiale. À chaque fois, la justification de telles démarches tombe sous le coup de l'évidence : «Parce que nous sommes les seuls dans la communauté... 36 ». Même si elles apparaissent naturelles au plus grand nombre, ces initiatives suscitent parfois des tensions entre de «simples » militants et d'autres, assurément plus

35. Becker H. S., « Notes on the concept of Commitment ", American Journal of Sociology, vol. 66, $\mathrm{n}^{\circ} 1,1960$, pp. 32-40. Je reprends ici la traduction de commitment choisie par Fillieule O., « Temps biographique, temps social et variabilité des rétributions », in Fillieule O. (dir.), Le Désengagement militant, Paris, Belin, 2005, p. 39.

36. Échange avec le principal leader de l'UPM, à l'issue d'une réunion durant laquelle des militants de son organisation tentèrent de venir en aide à trois femmes licenciées par l'un des fournisseurs de l'université de Grahamstown, juillet 2012. 
politisés. À la fin des années 2000, une assemblée du SECC voit, par exemple, s'opposer ceux qui attendent de l'organisation qu'elle initie un certain nombre d'activités dans la township, telle la création d'un potager, et ceux qui, défendant la conduite de projets approfondissant le combat contre le « capitalisme », estiment que ces demandes détournent la cause à des fins privées.

Quoi qu'il en soit, avant même d'être les porte-voix du mécontentement social, ces collectifs doivent donc être perçus comme des « organisations de la communauté »(community-based organisations), terminologie récurrente dans la parole militante. Ce constat transparaît avec évidence dans les entretiens, lorsque sont abordées les raisons de l'engagement protestataire. Ainsi, quand ils ont, par le passé, connu le monde des partis ou celui des syndicats, les activistes affirment s'être tenus à bonne distance de ces luttes intestines ou «pour les postes» qui scandent la vie de ces institutions. Ils mettent en revanche l'accent sur le fait que, aujourd'hui comme hier, leur engagement est subordonné au bien-être de la «communauté » ou des «pauvres» :

«Évidemment, avant je faisais partie de l'ANC... Et puis, j’allais aux réunions, et tout ça... Mais ça ne parlait que de politique, tu vois ? Il n'y avait rien sur la communauté... Rien pour la communauté... Moi, ça ne m’intéressait plus... Ici [au sein d'Abahlali], c'est différent. On est là pour la communauté... Ce que l'on fait, c'est pour elle. » (Entretien, Cape Town, 20 mai 2010)

«[...] le but du SECC, c'est d'aider les pauvres gens qui ne peuvent avoir... Qui ne savent pas comment faire face aux grosses factures d'Eskom [la compagnie d'électricité]... Parce que nous essayons de leur expliquer ce qui se passe... Comme ça, ils peuvent nous utiliser... Ils se servent de notre force [...] J'étais membre de l'ANC avant, depuis 1947... Jusqu'à ce que je quitte, en 2000 [Pourquoi ?] Parce qu'ils ne font pas attention à nous... Pas attention aux pauvres. » (Entretien, Johannesburg, 15 juillet 2009)

«Ceux qui se battent pour le TCC [Tembelible Crisis Committee] se battent pour la communauté. » (Entretien, Thembelihle, 22 avril 2015)

Cette subordination de l'engagement au bien de la communauté, systématiquement mise en avant, est même érigée en norme sociale de la vie dans la communauté :

«Quand nous allons devant les tribunaux ${ }^{37}$, parfois, nous demandons aux associations de taxis de nous y emmener gratuitement [Et

37. Les militants des organisations protestataires post-apartheid sont régulièrement poursuivis 
les taxis acceptent ?] Oui, ils acceptent. On va les voir en tant que Tembelible Crisis Committee et on leur dit : "Que faites-vous pour notre communauté ? Vous devez rendre quelque chose à la communauté" ». (Entretien, Thembelihle, 22 avril 2015)

L'engagement pour la communauté s'accompagne d'une responsabilité multiforme à son égard : d'une part, un mélange de redevabilité et de transparence dont témoigne l'usage récurrent de la notion d'accountability dans la parole militante ${ }^{38}$, d'autre part, une responsabilité sociale pouvant se traduire par des formes d'entrepreunariat socio-communautaire sur lesquelles je reviendrai :
« L'organisation essaie d'aider les gens... C'est ce qu'on fait, c'est ce qu'on doit faire, aider les gens et notamment les jeunes. On essaie de les tenir à distance de la rue et du crime... On leur apprend à se débrouiller. On les aide pour passer leur permis, tu vois ? On leur apprend quelles qualifications ils doivent avoir... En business... En informatique. On fait ça dans les réunions. Et moi, mon idée, c'est que l'organisation ouvre un centre pour les jeunes... Tu vois, ça, c'est à nous de le faire ". (Entretien avec un activiste d'Abablali baseMjondolo [AbM], Le Cap, 19 mai 2010)

Et ce «sens » de la responsabilité justifierait l'attente de contreparties, notamment sur le plan des loyautés :

«Les jeunes qui nous rejoignent... Certains, par exemple, veulent retourner à l'école. Alors, on essaie de les aider... On essaie d'aider la personne à faire un choix de carrière... On essaie de voir si elle peut obtenir une bourse [...] Comme ça, cette personne s'associe au SECC. Elle se dit : “J'étais fauché et voilà des gens qui m’ont aidé”... Ça aide la jeunesse et ça renforce l'organisation, tu vois ? » (Entretien, Soweto, 11 mai 2011)

On perdrait certainement beaucoup à ne voir, dans ces différents propos, qu'une forme d'idéalisation de l'engagement ou, plus simplement, la « mauvaise foi structurelle des militants 39 » recueillie par le chercheur. Le « travail

pour «trouble à l'ordre public » ou « destruction de la propriété », ce qui incite nombre de leurs dirigeants à déplorer une «criminalisation » du mécontentement social.

38. C'est ce dont rend bien compte la pratique du «report», qui conduit par exemple, au lendemain d'une entrevue avec la ministre provinciale du Logement, les militants du TCC à organiser une grande réunion sur un terrain vague, aux abords de la zone de logements informels. Pendant plus d'une heure, une poignée d'entre eux, armée d'un mégaphone, expose les conclusions du rendez-vous devant cent cinquante à deux cents personnes (Observation, Thembelihle, avril 2015).

39. Agrikoliansky É., "Carrières militantes et vocation à la morale : les militants de la LDH dans les années 1980 ", Revue française de science politique, vol. 51, n 1 et 2, février-avril 2001, p. 32 . 
de mise en scène de l'identité » auquel se prêtent les activistes interrogés révèle surtout avec précision les «catégories fondamentales » au travers desquelles ils pensent leurs pratiques; des catégories qui renseignent sur la construction de leur «identité publique », sur la manière dont ils perçoivent leur " position dans le monde social 40 » et, plus encore, au sein du quartier où ils vivent. Mais ces paroles et les actes qui leurs sont associés ne prennent pleinement sens que s'ils sont rapportés à un cadre particulier, celui qui nait de l'unification des dimensions physiques, sociales, culturelles et symboliques des quartiers populaires, et que saisit ce mot de tous les jours : la « communauté ».

\section{Le monde à part de la communauté}

S’ils sont, au cours de réunions publiques ou au fil d'entretiens, régulièrement présentés par leurs dirigeants et militants comme des "mouvements de pauvres pour les pauvres », les collectifs protestataires sont avant tout donnés à voir comme les porte-voix de la « communauté ». Ce faisant, ils se raccordent à une identité collective qui déborde la contestation postapartheid. Récurrente dans les médias, centrale dans la littérature administrative et politique et, plus généralement, dans la langue des producteurs de discours publics (élus nationaux et locaux en tête), la référence à la « communauté » innerve par ailleurs la parole des « gens ordinaires ». Le terme renvoie, en premier lieu, à la population d'un espace aux contours plus ou moins déterminés, un voisinage ou une rue le plus souvent. Mais au-delà, la référence à la « communauté » est une « mise en sens » et « en forme du social " ${ }^{41}$. Elle présuppose en outre l'existence de cet ensemble de croyances, de significations et de compréhensions (morales, émotionnelles, cognitives) partagées et incarnées que James Jasper désigne comme la culture d'un groupe ${ }^{42}$.

La communauté repose tout d'abord sur la conscience, commune à une majorité d'habitants des zones ouvrière et/ou pauvres d'Afrique du Sud, de vivre dans un monde à part et relativement cohérent. Une telle perception des choses - celle de former un tout au sein de la société - n'est pas sans fondement. Elle peut se nourrir du sentiment de relégation sociale et spatiale que ressentent ces individus et qui prend forme dans un discours reposant sur la classique opposition entre " eux et nous 43 ». Eux, ce sont alors tous ceux qui ne vivent pas dans ces quartiers, dans ces communautés; tous ceux qui auraient bénéficié de l'émancipation et du développement induits par la fin de l'apartheid; tous ceux qui, plus simplement, formeraient « le reste de la

40. Idem.

41. Vidal D., «Concevoir la communauté. L'efficacité d'une catégorie socio-spatiale au Brésil », in Monnet J. (dir.), Espace, temps et pouvoir dans le nouveau monde, Paris, Anthropos, 1996, p. 214.

42. Jasper J., The Art of Moral Protest: Culture, Biography and Creativity in Social Movements, Chicago, Chicago University Press, 1997.

43. Hoggarth R., La Culture du pauvre. Étude sur le style de vie des classes populaires en Angleterre, Paris, Les Éditions de minuit, 1970. 
société ». Cette vision du monde apparaît d'autant plus forte qu'elle s'objective dans ces frontières physiques que l'émergence de la démocratie libérale n'a pas menacées : ces kilomètres, déjà évoqués, qui s’immiscent entre, d'un côté, les townships (et, plus exactement, leurs quartiers les plus modestes, où les habitants disposent très rarement de moyens de locomotion), d'un autre, les quartiers résidentiels et les centres-villes; soit, en d'autres termes, tout ce qui tient les pauvres à distance de la concentration des emplois, des infrastructures de transport décentes, des centres commerciaux, des «bonnes » écoles et, plus globalement, des conditions d'une vie considérée comme «normale ». C'est donc une ligne de partage déjà présente dans l'ordonnancement d'une «partie de la vie sociale ordinaire 44 » que s'approprient les militants, notamment pour accuser leurs adversaires - les professionnels locaux et nationaux de la politique - d'avoir, pour la plupart, déserté les quartiers populaires pour s'installer dans les zones plus cossues des townships ou dans ces banlieues que l'on qualifiait autrefois de blanches et s'asseoir ainsi parmi ceux qui « mangent en premier 45 ».

Revendiquer une telle identité sociale - celle de représentante de la communauté - ouvre à la protestation l'accès à un véritable patrimoine, qui se décline en une histoire commune (celle de la lutte contre l'apartheid, notamment), des valeurs propres (une solidarité entre voisins présentée comme naturelle, une capacité de résistance aux gouvernants illégitimes, un sens de la débrouillardise, une certaine idée de l'égalité, etc.), des conditions d'existence partagées (quand bien même celles-ci varient d'une zone à l'autre de la township, cet espace étant loin d'être socialement homogène) et un large panel de « matériaux culturels 46 » (récits, symboles, styles vestimentaires et verbaux ou rituels, comme cette manière très spécifique de serrer la main de son interlocuteur dans les quartiers noirs). On pourrait évidemment soupçonner quelque stratégie dans cette façon qu'ont les meneurs de la protestation postapartheid de chercher à capter quelque chose qui la déborde ; une stratégie que dévoile, par exemple, l'intitulé de cet atelier organisé à Soweto en 2010 : «Comment devenir un leader dans sa communauté ?». Ne se glisse-t-elle pas également derrière cet effort régulier des militants pour convaincre de l'absence de distance sociale entre eux-mêmes et les populations qu'ils cherchent à mobiliser ? Tous, militants et habitants, seraient ainsi « dans le même bateau 47 », pour reprendre la formule choisie par l'un des collectifs du mécontentement. Et il est vrai que cette parfaite cö̈ncidence entre identité et représentation n'est en soi pas un leurre. On croise dans les rangs des organisations protestataires des

44. Tilly C., Tarrow S., Politique(s) du conflit. De la grève à la révolution, Paris, Presses de Sciences Po, 2008, p. 140.

45. Mandela Park Backyarders, "We are all in the same boat. Solidarity Statement with the Unemployed People’s Movement in Grahamstown », communiqué de presse du 13 janvier 2012.

46. Polleta F., Jasper J. M., « Collective identity and social movements ", Annual Review of Sociology, vol. 27, 2001, p. 285.

47. Mandela Park Backyarders, art. cit. 
« gens ordinaires », selon les mots de nombre de leurs dirigeants 48 : des jeunes gens sans emploi, des chômeurs de longue durée et d'âge mûr vivant parfois de l'économie informelle ou encore, des retraités assurant souvent l'existence de leurs enfants et petits-enfants avec leur seule pension. Certes, les individus les plus actifs, ceux qui assument le plus de responsabilités, ceux qui sont également amenés à parler au nom du collectif, ont en général un niveau d'instruction supérieur aux personnes qui composent la base sociale de l'organisation, même si leur trajectoire scolaire reste avant tout marquée par des renoncements ou des bifurcations imposées par des contingences financières. Mais au-delà de cet aspect, se dessine dans les rangs de la protestation un portrait social assez réussi d'une Afrique du Sud contemporaine où le chômage concerne, depuis le début des années 2000, près de $40 \%$ de la population active ${ }^{49}$, quand la pauvreté y est certainement la chose la mieux partagée. La majeure partie de ces militants connaît par ailleurs des conditions de logement ne la différenciant pas des publics qu'elle entend représenter : les membres d'Abahlali, au Cap et à Durban, vivent dans des cabanes en tôle, quand ceux du SECC habitent le plus souvent des quartiers frustes ayant connu des vagues de déconnexions dans les années 2000 et patientent - parfois depuis près de quinze ans - sur les listes d'attribution de maisons RDP. Cette situation tend à légitimer le fait que la protestation sociale postapartheid soit campée, tant par ses meneurs que par de simples et épisodiques militants, en mouvement porté par « les membres de la communauté ». En plus de nourrir l'affirmation/revendication d'un ancrage dans le paysage social des quartiers populaires, cette présentation des choses aide les militants à se penser en pauvres résistant au nom de leurs semblables. Cela n'est évidemment pas dénué d'enjeux dans la mesure où toute institution - et les organisations protestataires n'échappent pas à cette règle - tire en grande partie «son "pouvoir" de sa capacité à gérer [les] constructions identitaires [de ses membres] à l'aide de modèles plus ou moins explicites 50 ». Et il y a bien un modèle explicite de l'engagement qui, ici, s'impose et impose des mises en conformité comme autant de sources de légitimité dans la vie du quartier : celui du leader communautaire ou de l'activiste social.

48. L'ordinarité suggère que ces publics n'ont pas vocation à se révolter. Pas sans raison, du moins : c'est par nécessité que ces retraités, ces chômeurs de longue durée, ces parents isolés et ces jeunes sans emploi manifestent ; afin de résister à l'agression économique dont seraient victimes les pauvres d'Afrique du Sud.

49. Si, depuis le début de la décennie 2000, les chiffres officiels fournis par les autorités nationales situent le chômage aux alentours des 20-25\% de la population active, certaines institutions internationales, comme l'OCDE, avancent des taux bien plus élevés.

50. Dubar C., "Socialisation politique et identités partisanes : pistes de recherche », in CURAPP, L'Identité politique, Paris, Presses universitaires de France, 1994. 


\section{Des méritocraties locales 51 ?}

Le leadership communautaire et l'activisme social contribuent, au travers des activités qu'ils recouvrent, à réifier la communauté en la donnant à voir comme une entité dotée d'une puissance d'agir et d'une aptitude à traiter ses propres maux. Les positions sociales auxquelles ils sont associés résultent généralement d'une élection par les habitants d'une rue ou d'un quartier réunis au sein d'un comité, ou d'une présence à la tête d'une association ou d'un collectif localement influent. Partant, le leader communautaire, par exemple, est celui que l'on sollicite pour régler un différend entre voisins, pour obtenir une information ou un soutien dans une démarche administrative. C'est à sa porte que l'on tape en pleine nuit lorsqu'il s'agit de trouver au plus vite un véhicule pour transporter à l'hôpital une femme sur le point d'accoucher ${ }^{52} \ldots$ ou pour obtenir des boissons fraîches un jour de canicule ${ }^{53}$. C'est également à lui de s'assurer que «tout va bien » chez les voisins au lendemain de ces pluies torrentielles qui laissent régulièrement les quartiers pauvres dans des situations désastreuses ${ }^{54}$. Dans l'essai qu'il a consacré à Diepsloot, une localité secouée par de violentes manifestations en 2008 et 2009, Anton Harber décrit avec justesse la centralité de ces individus. Elle transparaît dans le récit de sa déambulation aux côtés d'une figure locale, la secrétaire de la section SANCO. Le lecteur découvre ainsi les multiples sollicitations dont la jeune femme fait l'objet tout au long de cette traversée de la township, comme celle de ce passant lui demandant d'intervenir auprès d'un garagiste qui refuse de lui rendre la batterie de son véhicule ${ }^{55}$. Le leader communautaire est donc un médiateur au sein du quartier, mais il peut également s'affirmer en intermédiaire régulier entre la population et les autorités politiques et administratives. On trouve, au sein des organisations protestataires, des individus dont le profil reprend assez nettement les principaux traits de cette rapide présentation ou, à tout le moins, des individus pouvant revendiquer un même « capital d'autochtonie 56 » (i.e. un capital social populaire et local articulé autour de relations de voisinage, de parenté ou d'amitié, d'une part, d'une réputation locale, d'autre part). Au cours d'entretiens, plusieurs militants se disent d'ailleurs «très connus » au sein de leur quartier, certains précisant même que cette notoriété est antérieure à leur entrée dans la protestation. C'est ce que confirme une analyse des parcours de femmes et d'hommes investis dans le mécontentement actuel. Elle met en évidence, chez ceux qui sont les plus visibles dans leur quartier, une propension à multiplier les engagements locaux («En tant que membre de la communauté, tu es toujours impliqué dans plein

51. Retière J.-N., « Autour de l'autochtonie. Réflexions sur la notion de capital social populaire », Politix, vol. 16, n 63, 2003, pp. 121-143.

52. Entretien avec le chairperson d'Abablali baseMjondolo Western Cape, 20 mai 2010, Kayelitsha (Le Cap).

53. Entretien avec une militante du TCC, 24 avril 2015, Thembelihle.

54. Entretien, 15 juillet 2009, Johannesburg.

55. Harber A., Diepsloot, Johannesburg E Cape Town, Jonathan Ball Publishers, 2011, p. 74.

56. Retière J.-N., art. cit. 
de choses dans le quartier ${ }^{57}$ ») et un investissement récurrent dans des formes d'entrepreneuriat sociocommunautaire, c'est-à-dire dans des actions relevant d'un certain don de soi et qui, au quotidien, sont menées au nom de l'intérêt de la communauté. Dans les années 2000, à Elsies River, dans l'agglomération du Cap, des membres de l'AEC apportent leur aide à ceux du civic (les deux organisations «partagent » certains militants) dans la distribution d'une soupe populaire placée sous la responsabilité de la mosquée locale. À Durban, dix ans auparavant, celle qui sera par la suite l'un des premiers visages féminins d'AbM est à l'origine de la création d'une crèche autogérée au milieu des baraquements de Kennedy Road. La reconnaissance qu'elle tire de cette initiative la conduit, au début de la décennie suivante, à être élue vice-présidente du comité de développement du campement, l'instance chargée de traiter les maux rencontrés au quotidien par les habitants et de négocier avec la municipalité. Et l'on prend plus encore la mesure de ces visibilités locales, qui se construisent et s'entretiennent presque au jour le jour, en se mettant dans les pas de l'un des meneurs de la contestation à Grahamstown depuis 2009. Ancien militant de l'Azanian People's Organisation (AZAPO) ${ }^{58}$, investi depuis de nombreuses années dans l'encadrement du football amateur (une activité qui, chaque week-end, concerne des dizaines de jeunes habitants des townships de la ville) et salarié depuis 2013 par une ONG spécialisée dans le «développement local », celui-ci arpente les quartiers noirs de sa ville du matin au soir ${ }^{59}$, vérifiant ainsi sa notoriété. Il passe le plus clair de son temps à s'assurer de la présence de tel ou tel à une prochaine réunion de l'UPM, « recrute » pour l'organisation quand l'occasion se présente, croise des leaders communautaires, s'enquiert du sort des jeunes qui vivent dans une décharge municipale, prend des nouvelles de voisins ou de connaissances, s'assied longuement sur les bords des terrains de football pour observer les progrès des adolescents et discuter éventuellement politique. Cet entremêlement des genres, des rôles, des identités, des intérêts et des réseaux, s'objective, là encore, dans des initiatives sociocommunautaires, à l'image de ce projet d'académie du football qu'il porte avec un autre militant UPM également responsable associatif. Construite au milieu d'une township, sur un terrain que les deux hommes espèrent obtenir de la municipalité (municipalité qu'ils contestent par ailleurs, en raison du retard pris dans le développement local), l'institution encadrerait les enfants à la sortie de l'école. Parallèlement, ce second militant, qui entraîne une équipe de footballeurs des townships, organise des veilles au sein de la communauté. Ses jeunes joueurs sont invités à y mener des actions collectives : s'assurer, par le biais de visites régulières, que les retraités ont un accès suffisant à l'eau, développer des jardins potagers dans les arrièrecours, mettre en place des soupes populaires, etc.

57. Entretien, Johannesburg, 15 juillet 2009.

58. L'AZAPO est une organisation politique fondée en 1978 et influencée par le Mouvement de la conscience noire de Steve Biko.

59. Observations réalisées en juillet 2012 et avril 2014. 
L'activité protestataire de ces individus s'insère donc dans une chaîne d'implications ou de dévouements (diachroniques et synchroniques) à des causes locales : associatives, civiques mais également politiques, comme le confirme la présence, dans les rangs de la protestation sociale, d'individus ayant fréquenté ou fréquentant des sections partisanes locales. Cette situation nourrit évidemment le capital social spécifique évoqué plus haut et c'est, à n'en pas douter, sur celui-ci que se construit la capacité de ces femmes et de ces hommes à mobiliser au sein de leur quartier, et ce presque quotidiennement depuis des années.

Nous nous sommes donc brièvement promenés sur les «bords » de la protestation ou, plus exactement, dans les marges de son acception la plus courante. Et il semble que l'on puisse, depuis cette périphérie, éclairer en partie ce qui se passe au cœur de l'activité protestataire, là où se diffuse l'adrénaline des marches et des invasions de terrains. S'y trouvent en effet des éléments de réponses à des questions aussi essentielles que "Comment la protestation tient-elle dans le temps ? ", "Qui manifeste ? » ou encore, "Comment manifeste-t-on?».

Arpenter les «bords» de la protestation offre en outre la possibilité de constater que les frontières de ce monde ne sont pas toujours tracées d'une main ferme. Leur indécision n'est pas un phénomène propre à l'Afrique du Sud, comme l'a montré Lilian Mathieu dans ses travaux sur l'espace des mouvements sociaux français 60 . Mais dans le cas sud-africain, les continuités avec la vie des quartiers populaires confèrent une consistance certaine à la contestation. Sa légitimité et, plus encore, son existence même apparaissent liées à son enracinement dans la communauté, véritable espace de références et de repères sociaux des collectifs militants. Derrière le geste protestataire, «[la révolte] laisse [ainsi] paraitre un ensemble de comportements, de convictions, de relations, de valeurs qui relèvent de l'expérience quotidienne ${ }^{61}$.

60. Mathieu L., L'Espace des monvements sociaux, Bellecombe-en-Bauges, Le Croquant, 2012.

61. Farge A., Revel J., Logiques de la foule. L'affaire des enlèvements d'enfants: Paris 1750, Paris, Hachette, 1988, p. 9. 